\title{
Angiogênese na Neoplasia Escamosa do Colo Uterino: Comparação entre dois Marcadores de Células Endoteliais
}

Angiogenesis in Squamous Cervical Neoplasia:

Comparative Study of two Endothelial Cells

Nilciza Maria de Carvalho Tavares Calux, Julisa Chamorro Lascasas Ribalta, João Norberto Stávale Jose Focchi, Edmund Chada Baracat, Geraldo Rodrigues de Lima

\section{RESUMO}

Objetivo: comparação entre a acurácia dos marcadores de células endoteliais anti-CD34 e anti-fator VIII em neoplasia cervical uterina, em lesões intra-epiteliais e no colo normal.

Métodos: os antígenos CD34 e ofator VIII foram identificados, por meio de técnicas de imunohistoquimica, em 18 fragmentos de neoplasia invasiva, 15 de lesão intra-epitelial de alto grau e 15 de baixo grau e 10 de colos normais, tidos como grupo controle. Fez-se a contagem dos vasos em 10 campos com aumento de 400X, na área de maior densidade vascular. $O$ total de vasos de cada caso resultou da soma dos 10 campos.

Resultados: as médias dos vasos corados com anti-CD34 no carcinoma invasivo, em lesão de alto grau, de baixo grau e no colo uterino normal foram 154, 135, 112 e 93 vasos, respectivamente. Com o uso do anti-fator VIII as médias das densidades vasculares foram 56, 44, 33 e 30 vasos, seguindo a mesma ordem das amostras. Lesões de alto grau e carcinomas invasivos do colo apresentaram médias de vasos maiores do que as de colos normais.

Conclusões: o emprego do anti-CD34 permite detecção de maior número de vasos do que o uso de anti-fator VIII. Confirmou-se a utilidade de ambos os marcadores na avaliação da angiogênese. A angiogênese diferencia lesões de baixo e alto grau, utilizando-se anti-fator VIII, o que não ocorre com anti-CD34.

PALAVRAS-CHAVE: Angiogênese. Colo: lesões pré-neoplásicas. Colo: carcinoma. Imunohistoquimica.

\section{Introdução}

Tumores malignos dependem da angiogênese local para proliferar, segundo estudos recentes ${ }^{1}$. A neo-angiogênese ocorre na transição do processo hiperplásico para neoplasia, portanto antes da formação tumoral propriamente dita. Este fato sugere que a indução da angiogênese constitui etapa importante na carcinogênese, também confirmado em estudos realizados em colo uterino ${ }^{2,3}$.

A neoformação vascular, além de ocorrer em

Departamento de Ginecologia da Universidade Federal de São Paulo - Escola Paulista de Medicina

Correspondência:

Nilciza Maria de Carvalho Tavares Calux

Rua França Pinto, 280/apto 82 - Vila Mariana

04016-001 - São Paulo - SP

Tel: (011) 5572-4970 neoplasias malignas, também o faz nos processos fisiológicos como, por exemplo, na maturação dos folículos ovarianos, na formação do corpo lúteo e no reparo do endométrio funcional. Surge, ainda, nos processos patológicos benignos como reparo de feridas, osteoartrite, em várias doenças oftálmicas e na reação imunológica ${ }^{4}$.

O mecanismo pelo qual os tumores malignos induzem e regulam a angiogênese ainda não é de todo conhecido. Em 1968, Greenblatt e Shubik $^{5}$, utilizando transplante de melanoma maligno, separado da rede vascular estromal do hospedeiro por fina membrana, demonstraram que fatores específicos produzidos pelas células tumorais induzem o desenvolvimento de novos vasos no hospedeiro. $O$ fator angiogênico tumoral, isolado pela primeira vez no começo da década de 1970, revelou ser mitogênico sobre as células endoteliais ${ }^{6}$. 
A partir de então, vários fatores angiogênicos foram descritos, dentre eles o fator de crescimento dos fibroblastos (variedade básica e ácida). Outros fatores angiogênicos, já bem identificados, são: a angiogenina, o fator de crescimento transformante $\alpha$, o fator de crescimento transformante $\beta$, o fator de crescimento endotelial produzido pelas plaquetas e o fator de necrose tumoral $\alpha$. O fator de permeabilidade vascular ou fator de crescimento do endotélio vascular é importante fator angiogênico na neoplasia cervical ${ }^{7,8}$.

Estudos recentes comprovam que a atividade angiogênica é determinada pelo equilíbrio entre a produção de fatores indutores e inibidores. $\mathrm{O}$ $\alpha$ interferon e o fator 4 plaquetário são elementos que podem coibir a angiogênese. São descritos ainda, como fatores inibidores, a trombospondina 1 e a angiostatina ${ }^{9}$.

A primeira evidência de que o número de vasos neoformados pode ser empregado como fator prognóstico foi observada no melanoma cutâneo humano. Descreve-se uma fase inicial, sem neovascularização, associada à ausência de metátases, seguindo-se um estágio com neovascularização crescente, que se correlaciona com o aumento do número de metástases ${ }^{10}$. Vários outros estudos confirmam a neoformação vascular como importante fator prognóstico do câncer de mama ${ }^{11}$, do carcinoma ovariano ${ }^{12}$ e do câncer do colo uterino ${ }^{13-15}$.

Entre os vários procedimentos descritos nos últimos anos para o estudo da angiogênese, assinalam-se os implantes tumorais em córnea de coelho, os implantes tumorais em membrana cório-alantóide de ovos de galinha e a cultura de células endoteliais ${ }^{4}$. Esses métodos são dinâmicos e possibilitam o estudo direto da angiogênese, entretanto, são de difícil execução na prática diária.

A mensuração da densidade vascular em câncer de mama, por meio da identificação imunohistoquímica do antígeno relacionado ao fator VIII das células endoteliais, foi sugerido por Weidner et al. ${ }^{11}$. A partir de então, a técnica imunohistoquímica para a identificação de vasos neoformados, utilizando a formação de complexo antígeno-anticorpo específico, vem sendo largamente utilizada por ser de fácil reprodutibilidade na maioria dos laboratórios e por apresentar resultados bastante satisfatórios.

Estudos com imunofluorescência e cultura de tecidos demonstram que células endoteliais sintetizam o antígeno relacionado ao fator VIII. A microscopia eletrônica evidencia o fator VIII também em plaquetas humanas e em megacarióci$\operatorname{tos}^{16}$. Por outro lado, Hollingsworth et al. ${ }^{12}$ deram preferência ao anti-CD34, entre os vários anticorpos testados para demonstrar a angiogênese no carcinoma ovariano.

Fina et al. ${ }^{17}$ mostraram a presença de antígeno CD34 em células endoteliais e sugerem que a molécula CD34 poderia participar da adesão celular tanto nas células endoteliais como nas progenitoras hematopoiéticas.

Também Schlingemann et al. ${ }^{18}$, investigando a precisa localização do antígeno CD34 nos vasos, observaram com o uso da microscopia eletrônica coloração para o antígeno mais forte na membrana próxima ao lúmen dos vasos, nos tecidos normais. Já nos tumores a identificação antigênica ocorre nos microprocessos, formados pela membrana celular do endotélio, voltada para o estroma.

Fina et al. ${ }^{17}$ chamaram a atenção para os diferentes comportamentos dos anticorpos antiCD34 e anti-fator VIII como marcadores vasculares, não só nos diversos tecidos como também dentro de um determinado tecido. Hollingsworth et al. ${ }^{12}$ e Tanigawa et al. ${ }^{19}$, estudando ovário e estômago, respectivamente, observaram maior número de vasos corados com o anticorpo anti-CD34 do que com o anti-fator VIII.

Compulsando a literatura pertinente, não encontramos qualquer estudo no qual se comparassem as diferenças de contagem entre antiCD34 e anti-fator VIII para avaliar a angiogênese da neoplasia de colo uterino, fato que nos motivou para esta pesquisa.

\section{Material e Métodos}

Foram selecionadas biópsias e peças cirúrgicas de colo uterino pertencentes a 58 pacientes atendidas no Departamento de Ginecologia da Universidade Federal de São Paulo - Escola Paulista de Medicina (UNIFESP - EPM), no período de maio de 1989 a abril de 1997. O estudo foi aprovado pela Comissão de Ética da UNIFESP.

Do total de 58 mulheres, 18 tinham diagnóstico anatomopatológico de neoplasia escamosa invasiva (grupo A) e 30 de neoplasia intra-epitelial, sendo 15 de alto grau (grupo B) e 15, de baixo grau (grupo C). As 10 pacientes restantes não apresentavam qualquer processo neoplásico, sendo assim consideradas como controle (grupo D).

As pacientes com neoplasia invasiva apresentavam a doença em estádio clínico I, conforme estadiamento preconizado pela Federação Internacional de Ginecologia e Obstetrícia (FIGO) ${ }^{20}$, sendo excluídas as pacientes com diagnóstico de adenocarcinoma, assim como aquelas com tratamento prévio por irradiação e /ou drogas 
antiblásticas. O grupo controle (D) foi constituído por pacientes submetidas a procedimento cirúrgico por lesão benigna.

O material, encaminhado para processamento imuno-histoquímico, foi constituído por duas lâminas de cada uma das 58 pacientes. A primeira foi preparada para a pesquisa de fator VIII e a segunda, para a do antígeno CD34. Para tanto, foram utilizados anticorpos primários, monoclonais, obtidos por imunização de animais de laboratório com antígenos humanos.

Anticorpos séricos de coelho (Rabbit antihuman Von Willebrand factor, Dako Polyclonal, Sta. Barbara, CA, USA - Lote 094 [021] Código M 0616), na diluição de 1:16.000, foram utilizados para a detecção do fator VIII.

Para identificar as células que expressavam o marcador CD 34 com CD34 empregamos anticorpos séricos de camundongos (Mouse antihuman CD34, Novocastra, clone Q B End 10, lote 512), na diluição de 1:50.

Para ambos os conjuntos usaram-se anticorpos secundários a partir do método Estreptoavidina-biotina-peroxidase (Strepto ABC, Dako, Código KO 492 lote 105 [102]), na diluição de 1:500.

As células endoteliais, assim tratadas, adquiriram coloração acastanhada com ambos os anticorpos, o que permitiu identificá-las (Figura 1). Simultaneamente realizou-se um "controle positivo", para a avaliação da eficácia do anticorpo primário, utilizando-se, para tanto, espécimes teciduais de amigdalas humanas.

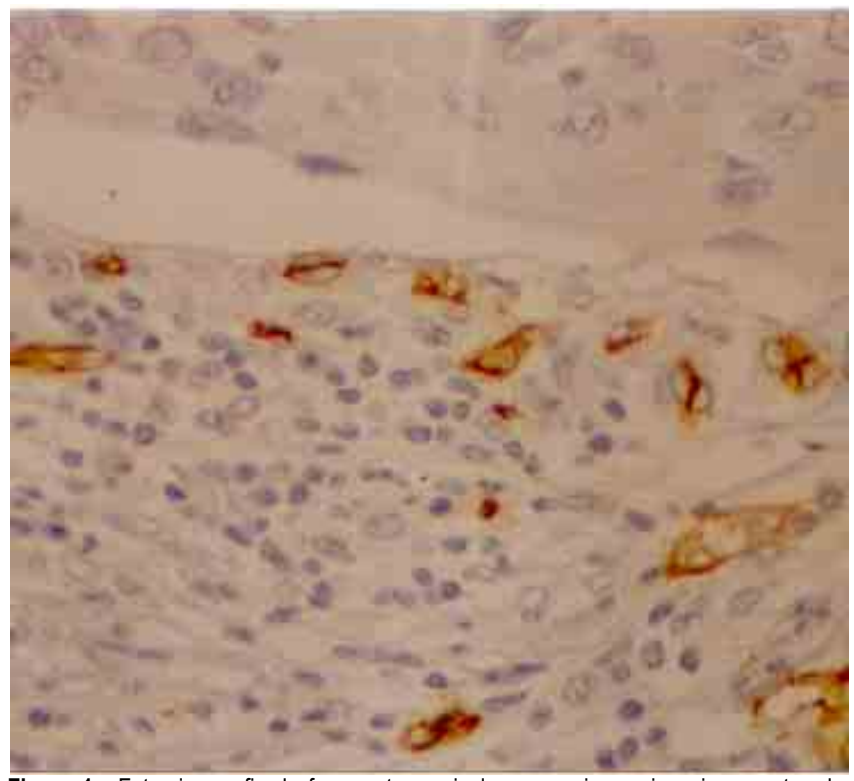

Figura 1 - Fotomicrografia de fragmento cervical com carcinoma invasivo, mostrando imunoexpressão do CD34 (400X).

Para a contagem dos vasos utilizou-se microscópio de luz com objetivas de 10X e 40X e ocu- lares de 10X, perfazendo aumentos de 100X e 400X, respectivamente. A objetiva de menor aumento foi usada para selecionar, nos casos do grupo controle e naqueles com neoplasia intra-epitelial, a área estromal subjacente ao epitélio normal ou atípico com maior densidade vascular ${ }^{3}$. Nos casos com neoplasia invasiva o mesmo recurso foi empregado para localizar a área mais vascularizada da interface tumor-córion ${ }^{11}$. Selecionada a área em cada caso, procedeu-se à contagem dos vasos em aumento de 400X.

Estabeleceu-se que o número total de vasos, obtidos em cada caso, seria o resultado do somatório do número de vasos contados em cada um dos 10 campos histológicos avaliados. Convencionou-se, ainda, considerar como unidade vascular a presença obrigatória de células coradas em castanho margeando o espaço luminal. Os vasos parcialmente identificados, não contidos por inteiro nos campos analisados, não foram computados. A contagem foi realizada por leitura direta nos aumentos citados, sem auxílio de qualquer dispositivo.

Dois observadores independentes, concomitantemente e obedecendo aos critérios citados, procederam à leitura das lâminas e coleta dos dados. Somente quando houve a concordância de ambos, o elemento identificado foi considerado como vascular.

Em virtude da natureza da variável, contagem do número de vasos, usamos para avaliação estatística não-paramétrica o teste de Wilcoxon para médias não-independentes e o teste de Kruskal-Wallis para médias independentes, complementado pelo teste de comparações múltiplas sempre que necessário.

O nivel de significância foi sempre igual ou menor do que 0,05 (5\%).

\section{Resultados}

Os resultados obtidos na contagem dos elementos vasculares utilizando-se dois marcadores imuno-histoquimicos (anti-CD34 e anti-fator VIII) estão expostos nas Tabelas 1 e 2 . A média dos vasos corados com anti-CD34 no carcinoma invasivo, em lesão de alto grau, de baixo grau e no colo uterino normal foi de 154, 135, 112 e 93 vasos, respectivamente, ao passo que com o uso do antifator VIII os números foram 56, 44, 33 e 30 vasos, seguindo a mesma ordem das amostras.

A média de vasos foi maior nos casos corados com os anticorpos anti-CD34 do que a obtida com anti-fator VIII (teste de Wilcoxon: $p<0,05$ ). Quando comparamos os quatro grupos (A, B, C e D) 
corados com o marcador vascular anti-CD34 entre si, usando o teste de Kruskal-Wallis complementado com o teste de comparações múltiplas (Tabela 1), notamos que o número de vasos dos casos normais não difere de maneira significante daquele das portadoras de neoplasia intra-epitelial de baixo grau. Por outro lado, quando se comparou o grupo sem neoplasia com os grupos com neoplasia intra-epitelial de alto grau e com carcinoma invasivo, o número de vasos nas epiteliopatias relacionadas com malignidade foi maior, com comprovada significância estatística.

Tabela 1 - Distribuição dos valores correspondentes ao número de vasos avaliados nos carcinomas invasivos de colo uterino $(A)$, lesões intra-epiteliais de alto grau $(B)$ e de baixo grau $(C)$ e colos uterinos normais $(D)$ utilizando-se técnica imuno-histoquímica para identificação de estruturas vasculares com anti-CD34, médias e resultado da estatística.

\begin{tabular}{|c|c|c|c|c|}
\hline Caso $\left(n^{0}\right)$ & Grupo A & Grupo B & Grupo C & Grupo D \\
\hline 1 & 161 & 128 & 121 & 109 \\
\hline 2 & 160 & 131 & 98 & 65 \\
\hline 3 & 128 & 125 & 81 & 97 \\
\hline 4 & 168 & 141 & 128 & 96 \\
\hline 5 & 131 & 157 & 91 & 110 \\
\hline 6 & 107 & 132 & 117 & 67 \\
\hline 7 & 219 & 143 & 136 & 106 \\
\hline 8 & 147 & 112 & 95 & 102 \\
\hline 9 & 175 & 131 & 105 & 80 \\
\hline 10 & 119 & 128 & 128 & 96 \\
\hline 11 & 190 & 139 & 90 & \\
\hline 12 & 203 & 135 & 151 & \\
\hline 13 & 107 & 127 & 129 & \\
\hline 14 & 153 & 121 & 95 & \\
\hline 15 & 128 & 168 & 112 & \\
\hline 16 & 209 & & & \\
\hline 17 & 142 & & & \\
\hline 18 & 124 & & & \\
\hline Média & 154 & 135 & 112 & 93 \\
\hline \multicolumn{2}{|c|}{ Teste de Kruskal-Wallis } & \multicolumn{2}{|c|}{$\mathrm{H}_{\text {crit }}=7,815$} & \\
\hline \multicolumn{2}{|c|}{$H_{\text {calc }}=29,586^{*}$} & \multicolumn{2}{|c|}{$H_{\text {corr }}=29,628^{*}$} & \\
\hline \multicolumn{5}{|c|}{ Teste de Comparações Múltiplas } \\
\hline \multicolumn{2}{|c|}{ Pares } & VADMP & DMS & \\
\hline & $\begin{array}{l}O, C) \\
O, B) \\
, A) \\
C B \\
, A) \\
B, A)\end{array}$ & $\begin{array}{l}0,50 \\
6,40 \\
1,76 \\
5,90 \\
1,26 \\
5,36\end{array}$ & $\begin{array}{l}18,19 \\
18,19 \\
17,57 \\
16,27 \\
15,58 \\
15,58\end{array}$ & \\
\hline
\end{tabular}

VAMDP: Valor absoluto da diferença entre as médias dos postos; DMS: diferença mínima significante;

* significante; N.S.: não significante
Tabela 2 - Distribuição dos valores correspondentes ao número de vasos avaliados nos carcinomas invasivos de colo uterino (A), lesões intra-epiteliais de alto grau (B) e de baixo grau $(C)$ e colos uterinos normais $(D)$ utilizando-se técnica imuno-histoquímica para identificação de estruturas vasculares com anti-fator VIII, médias e resultado da estatística.

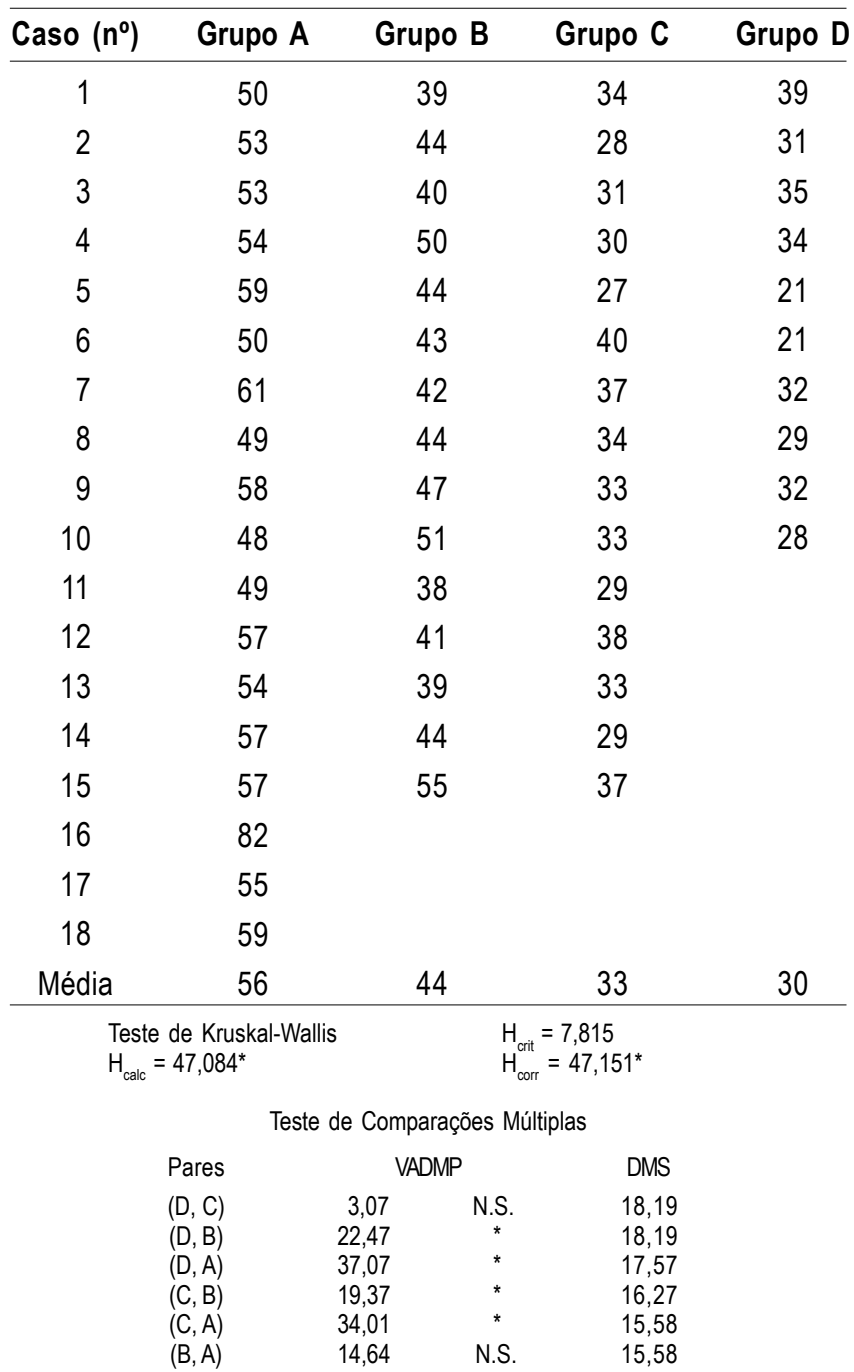

VAMDP: Valor absoluto da diferença entre as médias dos postos; DMS: diferença mínima significante;

* significante; N.S.: não significante

Considerando ainda a técnica imunohistoquímica para CD34 e comparando o número de vasos contados nos grupos A, B e C, evidenciase que o grupo $\mathrm{C}$, ou seja, casos com neoplasia intra-epitelial de baixo grau, não apresentou diferença estatística significante quando comparado ao grupo B (neoplasia intra-epitelial de alto grau). Entretanto, quando a comparação é feita entre os grupos C (lesão de baixo grau) e A (carcinoma invasivo), a diferença é significante. Finalmente, quando se compara o número de vasos contados no grupo B (lesão de alto grau) com o do grupo A (carcinoma invasivo) também não se observa diferença.

Utilizando-se a coloração anti-fator VIII (Tabela 2), verificou-se, mais uma vez, que o grupo D 
(grupo controle), quando comparado aos grupos A, $\mathrm{B}$ e $\mathrm{C}$, só é diferente dos grupos A (carcinoma invasivo) e B (lesão de alto grau), sendo pois semelhante ao $\mathrm{C}$ (lesão de baixo grau) (teste de Kruskal-Wallis: V = 7,8).

Os exemplares de neoplasia intra-epitelial de baixo grau (C) possuem número de vasos menor do que os encontrados na neoplasia intraepitelial de alto grau (B) e na neoplasia invasora (A). Não ocorre diferença quando comparamos o número de vasos dos grupos $\mathrm{A}$ e $\mathrm{B}$.

\section{Discussão}

Apesar de opiniões contrárias, como as de Kainz et al. ${ }^{14}$, alguns autores demonstraram que, no carcinoma invasivo de colo uterino, a densidade vascular aumentada está relacionada com pior prognóstico ${ }^{13,15}$.

A neoformação vascular tem sido, ainda, quantificada nas neoplasias intra-epiteliais, visando estabelecer quando realmente esse processo tem início e, talvez, transportar o conceito vindo dos estudos com carcinomas invasivos, para os quais se pode inferir que quanto maior o número de vasos, mais agressiva seria a neoplasia. Parâmetros para decisões terapêuticas e de seguimento estariam assim adequadamente fundamentados, uma vez que lesões pré-neoplásicas intra-epiteliais, ditas de alto grau, podem progredir.

Já Ziche e Gullino ${ }^{21}$ em experimentos in vitro demonstraram que a capacidade angiogênica ocorre durante a transformação neoplásica, firmando a hipótese de que, quando células normais têm capacidade de formar novos vasos, possuem também maior risco de transformação neoplásica, ratificando o que Stafl e Mattingly ${ }^{22}$ evidenciaram no colo uterino, ou seja, as mudanças vasculares precedem as alterações histopatológicas.

No colo uterino, o fator VIII tem sido com freqüência utilizado na pesquisa de fatores prognósticos para o carcinoma espinocelular. Na avaliação da literatura, concernente ao emprego de anticorpos anti-CD34 em colos uterinos, encontramos o estudo de Miettinen et al. ${ }^{23}$ realizado em colos normais, descrevendo a identificação de vasos e de algumas células estromais, pela coloração acastanhada dessa prova imuno-histoquímica.

Observamos, ao comparar os valores encontrados nas avaliações de vasos marcados com anticorpos anti-CD34 e os corados com o anti-fator VIII, que o primeiro é capaz de evidenciar maior numero de vasos do que o segundo, tanto em lesões de baixo ou alto grau, quanto em carcinomas invasivos ou no colo uterino normal.

Os valores menores computados com o uso do anti-fator VIII, comparados aos do anti-CD34, também foram notados por Hollingsworth et al. ${ }^{12}$ ao estudar a angiogênese tumoral em carcinoma ovariano em estádio avançado. $\mathrm{Na}$ contagem de microvasos com anti-CD34 e anti-fator VIII em carcinomas gástricos, o número obtido com o primeiro corante foi aproximadamente três vezes maior que o notado com o segundo anticorpo ${ }^{19}$.

Acreditamos que a diferença numérica dos vasos seja conseqüência da presença de estruturas vasculares não coradas, no campo das lâminas preparadas com anti-fator VIII e, portanto, excluídas da contagem. Por outro lado, poder-se-ia dizer que, nas lâminas coradas pelo anti-CD34, foram contadas estruturas não vasculares, já que esse marcador cora também fibroblastos. Mais uma vez, acreditamos que esta falta não ocorre quando se tem o cuidado de cotejar apenas estruturas que têm lúmen. Por outro lado, vasos linfáticos não são corados pelo anti-CD34, nem pelo anti-fator VIII, sendo portanto excluídos de falsa avaliação ${ }^{23}$.

Ao analisar nossos resultados nos quatro grupos A, B, C e D, nas lâminas coradas com antifator VIII, notamos que o comportamento da lesão de baixo grau é semelhante ao do colo normal. Notamos ainda que o número de vasos aumenta a partir da lesão de alto grau, até se tornar semelhante ao do carcinoma invasivo. Isto nos leva a concluir que a neoformação vascular acontece nas lesões de alto grau, ou seja, nas formas préinvasivas mais próximas do desencadear da invasão.

Todos os trabalhos referentes à angiogênese e colo uterino usam o anti-fator VIII para a quantificação dos vasos, e nossos resultados estão de acordo com os de Smith-McCune e Weidner ${ }^{3}$, Guidi et al. ${ }^{8}$ e Ravazoula et al. ${ }^{2}$. Esses autores asseguram que a angiogênese começa na fase pré-invasiva e pode ser importante evento na conversão do epitélio normal a neoplásico.

Por outro lado, Abulafia et al. ${ }^{24}$ não encontraram maior número de vasos na neoplasia intraepitelial, ao passo que Soini et al. ${ }^{25}$ embora sem visualizar maior quantidade de vasos na lesão préinvasiva, demonstraram atividade proliferativa mais intensa nas células endoteliais de lesões NIC II e III em comparação aos condilomas e NIC I, valendo-se da positividade do antígeno nuclear de proliferação celular na célula endotelial como marcador da proliferação vascular.

Quando analisamos nossos resultados nas lâminas coradas pelo anti-CD34 notamos que, tal como ocorreu com o anti-fator VIII, a neovascularização aconteceu realmente a partir da lesão de alto grau até a lesão francamente in- 
vasora.

Com a coloração anti-CD34 notamos, entretanto, novo pormenor que nos sinaliza uma discreta tendência de aumento da angiogênese nas lesões de baixo grau (grupo C), já que o número de vasos contados nesse grupo não foi estatisticamente diferente do número de vasos encontrados no grupo B (neoplasia intra-epitelial de alto grau), diferenciando-se apenas do grupo A (carcinoma invasivo).

Esse achado pode ser explicado pelo estudo de Schlingemann et al. ${ }^{18}$ em cultura de células endoteliais de veia umbilical humana, onde a expressão de CD34 foi preferencialmente encontrada em células com aparência morfológica daquelas em migração, sugerindo que o CD34 é um marcador citológico endotelial no início do processo dinâmico da angiogênese. Vasos seriam corados desde o inicio da neovascularização e não somente aqueles já formados. Somos assim induzidos a afirmar que o anti-CD34 é mais sensivel que o anti-fator VIII, traduzindo-se em melhor marcador funcional do que apenas morfológico.

Reforçam esta assertiva as observações realizadas por Guidi et al. ${ }^{8}$ ao detectar, em fragmentos de mucosa cervical com alterações epiteliais benignas e de baixo grau, leves e moderados niveis de expressão do RNA mensageiro do fator de permeabilidade vascular, reconhecido como um dos importantes fatores angiogênicos. Afirmam, esses autores, que tais resultados estariam indicando resposta a fenômeno reativo fisiológico, ou reação inflamatória à agressão mórbida ou, ainda, processo epitelial neoplásico em início de progressão.

Outra ilação possivel, observada entre os valores de anti-CD34, dos grupos com neoplasia intra-epitelial de baixo (C) e de alto grau (B), seria: a falta de diferença entre os grupos $\mathrm{B}$ e $\mathrm{C}$ estaria traduzindo heterogeneidade de agentes desencadeadores do processo hiperplásico (HPV 6, $11,16,18$ e outros) e, quiçá, de tendências prognósticas diferentes.

Apesar de a angiogênese diferenciar lesão de baixo e alto grau, utilizando-se o anti-fator VIII, o mesmo não acontece quando empregamos o antiCD34. Isso nos faz acreditar que talvez fosse interessante associar outros marcadores prognósticos, em especial a identificação do tipo viral e a quantificação da carga viral para o papilomavirus humano. Assim, uma lesão só teria potencial para malignidade se tivesse elevado número de vasos neoformados, HPV de alto risco e com alta carga viral. Mais uma vez afiançam esta hipótese os trabalhos de Guidi et al. ${ }^{8}$ anteriormente citados.

Outra possibilidade a ser aventada poderia ser que o grupo B (lesão intra-epitelial de alto grau), por sua composição, integrando as anteriormente chamadas displasias moderadas, displasias graves e carcinoma in situ, seria também heterogêneo. Estariamos assim reunindo em um só grupo processos proliferativos de comportamentos diferentes e probabilidades distintas de evolução e / ou de regressão.

Nosso estudo permite-nos supor que eventuais enzimas que rompem a membrana basal dos vasos, no início da neovascularização, seriam as mesmas capazes de romper a membrana basal do epitélio e assim facilitar o processo de invasão. Pesquisas prospectivas deverão ser desenvolvidas para que esse tópico da biologia tumoral seja esclarecido.

\section{SUMMARY}

Purpose: to compare the efficiency of anti-factor VIII and anti-CD34 antibodies as vascular makers in cervical cancer, in cervical intraepithelial neoplasia and in normal cervix. Methods: using an immunohistochemical method, factor VIIIrelated antigen and leukocyte antigen CD34, we performed microvascular counts in 18 squamous cell carcinomas, in 15 cervical high-grade intraepithelial neoplasia, in 15 lowgrade intraepithelial lesions and in 10 normal cervices. Using light microscopy we counted microvessels per $400 X$ field in the most active areas of neovascularization with higher microvessel density in each case.

Results: the average of microvessels stained with anti-CD34 in invasive carcinoma, high-grade intraepithelial lesions, low-grade intraepithelial lesions and in the normal cervices was 154, 134, 112 and 93, respectively. When we used antifactor VIII the average was 56, 44, 33 and 30 vessels, following the same order. High-grade intraepithelial lesions and invasive carcinomas showed greater means number of vessels than normal tissue.

Conclusions: the use of anti-CD34 allowed the detection of a greater number of vessels when compared to anti-factor VIII. However, we could observe that anti-factor VIII staining was able to significantly discriminate high-grade from low-grade lesions.

KEY WORDS: Angiogenesis. Cervix: precursor lesions. Cervix: carcinoma. Immunohistochemistry.

\section{Referências}

1. Folkman J, Watson K, Ingber D, Hanahan D. Induction of angiogenesis during the transition from hyperplasia to neoplasia. Nature 1989; 339:58-61. 
2. Ravazoula P, Zolota V, Hatjicondi O, Sakellaropoulos G, Kourounis G, Maragoudakis ME. Assessment of angiogenesis in human cervical lesions. Anticancer Res 1996; 16:3861-4.

3. Smith-McCune KK, Weidner N. Demonstration and characterization of the angiogenic properties of cervical dysplasia. Cancer Res 1994; 54:800-4.

4. Knighton DR, Fiegel VD, Phillips GD. The assay of angiogenesis. Prog Clin Biol Res 1991; 365:2919.

5. Greenblat M, Shubik P. Tumor angiogenesis: transfilter diffusion studies in the hamster by transparent chamber technique. J Natl Cancer Inst $1968 ; 41: 111-6$.

6. Folkman J, Merler E, Abernathy C, Williams G. Isolation of a tumor factor responsible for angiogenesis. J Exp Med 1971; 133:275-88.

7. Folkman J, Klagsbrun M. Angiogenic factors. Science $1987 ; 235: 442-7$.

8. Guidi AJ, Abu-Jawdeh G, Berse B, et al. Vascular permeability factor (vascular endothelial growth factor) expression and angiogenesis in cervical neoplasia. J Natl Cancer Inst 1995; 87:1237-45.

9. Hanahan D, Folkman J. Patterns and emerging mechanisms of the angiogenic switch during tumorigenesis. Cell 1996; 86:353-64.

10.Srivastava A, Laidler P, Hughes LE, Woodcock J, Shedden EJ. Neovascularization in human cutaneous melanoma: a quantitative morphological and Doppler ultrasound study. Eur J Cancer Clin Oncol 1986; 22:1205-9.

11.Weidner N, Semple JP, Welch WR, Folkman J. Tumor angiogenesis and metastasis: correlation in invasive breast carcinoma. N Engl J Med 1991; 324:1-8.

12. Hollingsworth HC, Kohn EC, Steinberg SM, Rothenberg ML, Merino MJ. Tumor angiogenesis in advanced stage ovarian carcinoma. Am J Pathol 1995; 147:33-41.

13.Bremer GL, Tiebosch AT, van der Putten HW, Schouten HJ, de Haan J, Arends JW. Tumor angiogenesis: an independent prognostic parameter in cervical cancer. Am J Obstet Gynecol 1996; 174:126-31.

14.Kainz C, Speiser P, Wanner C, et al. Prognostic value of tumour microvessel density in cancer of the uterine cervix stage IB to IIB. Anticancer Res $1995 ; 15: 1549-51$.
15.Wiggins DL, Granai CO, Steinhoff MM, Calabresi P. Tumor angiogenesis as a prognostic factor in cervical carcinoma. Gynecol Oncol1995; 56:3536.

16.Piovella F, Nalli G, Malamani GD, et al. The ultrastructural localization of factor VIII - antigen in human platelets, megakaryocytes and endothelial cells utilizing a ferritin-labelled antibody. Br J Haematol 1978; 39:209-13.

17.Fina L, Molgaard HV, Robertson D, et al. Expression of the CD34 gene in vascular endothelial cells. Blood 1990; 75:2417-26.

18.Schlingemann RO, Rietveld FJR, de Waal RM, et al. Leukocyte antigen CD34 is expressed by a subset of cultured endothelial cells and on endothelial abluminal microprocesses in the tumor stroma. Lab Invest 1990; 62:690-6 .

19. Tanigawa N, Amaya H, Matsumura M, et al. Extent of tumor vascularization correlates with prognosis and hematogenous metastasis in gastric carcinomas. Cancer Res 1996; 56:26716.

20.FIGO Committee on Gynecologic Oncology. Modifications in the staging for stage vulvar and stage I cervical cancer. Int $\mathrm{J}$ Gynaecol Obstet 1995; 50:215-6.

21.Ziche M, Gullino PM. Angiogenesis and neoplastic progression in vitro. J Natl Cancer Inst 1982; 69:483-7.

22.Stafl A, Mattingly RF. Angiogenesis of cervical neoplasia. Am J Obstet Gynecol 1975; 121:84552.

23. Miettinen M, Lindenmayer AE, Chaubal A. Endothelial cell markers CD31, CD34, and BNH9 antibody to $\mathrm{H}$ - and $\mathrm{Y}$-antigens: evaluation of their specificity and sensitivity in the diagnosis of vascular tumors and comparison with von Willebrand factor. Mod Pathol 1994; 7:82-90.

24.Abulafia O, Triest WE, Sherer DM, Hansen CC, Ghezzi F. Angiogenesis in endometrial hyperplasia and stage I endometrial carcinoma. Obstet Gynecol 1995; 86:479-85.

25.Soini Y, Pöllänen R, Kemppainen S, Pääkko P, Lehto VP. The association of vascular proliferation with HPV status and epithelial PCNA positivity in cervical intraepithelial lesions. APMIS 1996; 104:183-90. 